\title{
Carrier-envelope phase dependent high-order harmonic generation with a high-repetition rate OPCPA-system ${ }^{\star}$
}

\author{
Piotr Rudawski ${ }^{1, a}$, Anne Harth ${ }^{1}$, Chen Guo $^{1}$, Eleonora Lorek ${ }^{1}$, Miguel Miranda $^{1}$, Christoph M. Heyl ${ }^{1}$, \\ Esben W. Larsen ${ }^{1}$, Jan Ahrens ${ }^{2}$, Oliver Prochnow ${ }^{2}$, Thomas Binhammer ${ }^{2}$, Uwe Morgner ${ }^{3}$, \\ Johan Mauritsson ${ }^{1}$, Anne L'Huillier ${ }^{1}$, and Cord L. Arnold ${ }^{1}$ \\ 1 Department of Physics, Lund University, P.O. Box 118, 22100 Lund, Sweden \\ 2 VENTEON Laser Technologies GmbH, Hertzstrasse 1b, 30827 Garbsen, Germany \\ 3 Institut of Quantum Optics, Leibniz Universität Hannover, Welfengarten 1, 30167 Hannover, Germany
}

Received 30 July 2014 / Received in final form 16 December 2014

Published online 12 March 2015

(c) The Author(s) 2015. This article is published with open access at Springerlink.com

\begin{abstract}
We study high-order harmonic generation with a high-repetition rate $(200 \mathrm{kHz})$, few-cycle, driving laser, based on optical parametric chirped pulse amplification. The system delivers carrier-envelope phase stable, $8 \mathrm{fs}, 10 \mu \mathrm{J}$ pulses at a central wavelength of $890 \mathrm{~nm}$. High-order harmonics, generated in a high-pressure Ar gas jet, exhibit a strong CEP-dependence over a large spectral range owing to excellent stability of the driving laser pulses. This range can be divided into three spectral regions with distinct CEP influence. The observed spectral interference structures are explained by an analytical model based upon multiple pulse interferences.
\end{abstract}

\section{Introduction}

The development of attosecond science strongly relies on the progress of ultrafast laser sources. Over more than twenty years, chirped pulse amplification (CPA) systems based on Titanium:Sapphire (Ti:Saph) technology have been used to drive XUV attosecond sources at repetition rates up to a few $\mathrm{kHz}$. Optical parametric chirped pulse amplification (OPCPA) systems $[1,2]$ are based on three-wave mixing in a nonlinear crystal, using as input waves, a stretched broadband seed pulse and narrowband pump pulse of matched duration. The seed pulse is amplified, while an idler pulse is generated. The current limitations in conventional amplifiers, such as gain-narrowing and thermal lensing, are avoided in parametric amplification. OPCPA technology allows for the amplification of few-cycle pulses with TW peak power [3] at low repetition rates and is particularly advantageous at high-repetition rate from hundreds of $\mathrm{kHz}$ [4-6] to few $\mathrm{MHz}$ [7-9]. Recently, OPCPA systems producing CEP-stable few-cycle pulses at high-repetition rate have been successfully used for high-order harmonic generation (HHG). Tünnermann and coworkers approached the single attosecond pulse

\footnotetext{
* Contribution to the Topical Issue "X-ray Generation from Ultrafast Laser", edited by Germán J. de Valcárcel, Luis Roso and Amelle Zaïr.

a e-mail: piotr.rudawski@fysik.1th.se
}

limit [10] and generated high-order harmonics in Ar with high average power [11].

In this work, we present a HHG source driven by a compact OPCPA laser producing CEP-stable $8 \mathrm{fs}, 10 \mu \mathrm{J}$ pulses, at $890 \mathrm{~nm}$ central wavelength, and $200 \mathrm{kHz}$ repetition rate [6]. To achieve sufficiently high intensity for efficient HHG in Ar, the laser beam is tightly focused $(f=5 \mathrm{~cm})$ into a high pressure effusive Ar gas jet. The generated HHG spectra exhibit a strong CEP-dependence covering the spectral region from 25 to $45 \mathrm{eV}$.

CEP-dependent effects in HHG have been observed in previous works [10,12-17], but have often been limited to specific spectral regions, such as the cut-off $[10,12,14]$ or the region between consecutive harmonic spectral peaks $[15,16]$. The interpretation of the observed CEP effects vary from quantum path interferences to interference between consecutive attosecond pulses. In the latter case, several effects have been discussed: the number of interfering pulses [13], the variation of timing between these pulses as well as the phase difference between consecutive pulses. Here, we develop a simple model consisting in coherently adding the spectra from the number of emitted attosecond pulses, which is estimated to be three in the present experiment, taking into account the variation of the spectral phase with the laser CEP. The good agreement between the results of this model and the experimental data indicates that the physics of the interference structures is contained in the variation of the spectral 


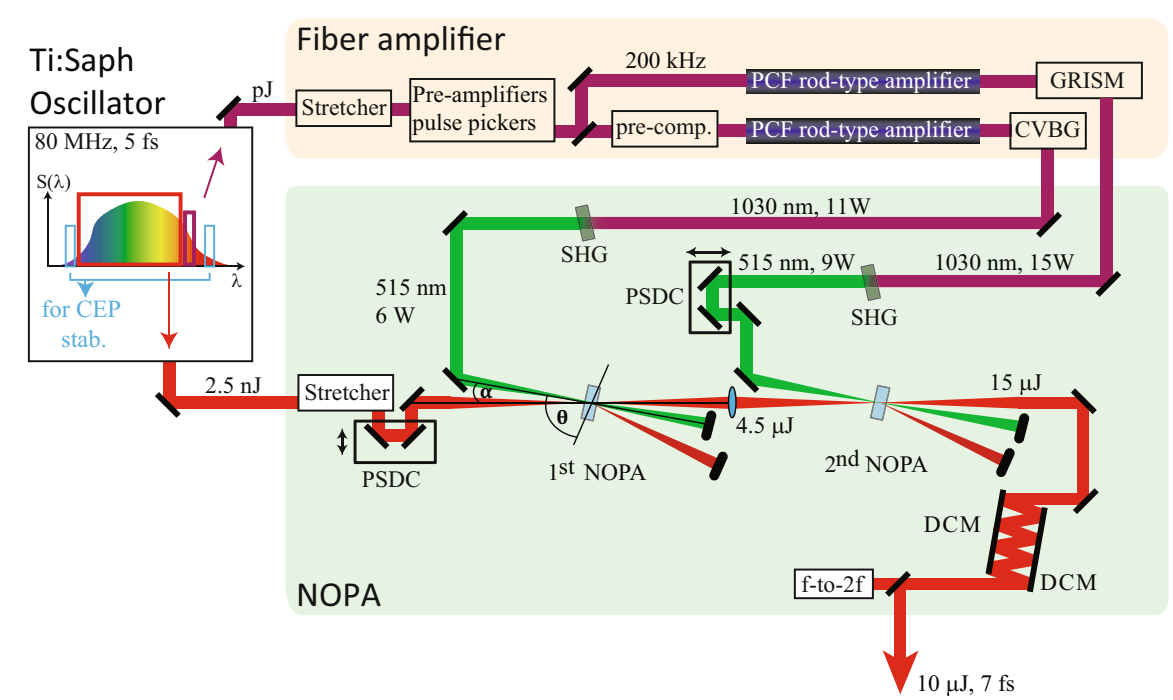

Fig. 1. The OPCPA system; PCF - photonic crystal fiber, CVBG - chirped volume Bragg grating, SHG - second harmonic generation in BBO crystals, PSDC - pump-signal delay control, NOPA non-collinear optical parametric amplification in BBO crystals, $\alpha$-non-collinear angle, $\theta$ - phase-matching angle, DCM - double chirped mirror.

phase of consecutive attosecond pulses and not only in the number of interfering pulses.

The OPCPA system and the HHG source are described in Sections 2 and 3. The CEP-dependent harmonic spectra are presented in Section 4 and interpreted in Section 5.

\section{Experimental setup: OPCPA}

The HHG setup is driven by an OPCPA system [6], sketched in Figure 1. The system comprises three main units: a broadband Ti:Saph oscillator (VENTEON), a pumping laser based on an Yb-doped fiber amplifier, and a two-stage Non-collinear Optical Parametric Amplifier (NOPA). The Ti:Saph oscillator produces an octave- spanning spectrum, supporting CEP stable pulses with duration below $5 \mathrm{fs}$. The rms phase noise, corresponding to a power spectral density integrated from $3 \mathrm{~Hz}$ to $1 \mathrm{MHz}$, is measured to be below $80 \mathrm{mrad}$ [18]. A small part of the oscillator spectrum around $1030 \mathrm{~nm}$ serves as seed for the fiber laser, whereas the main part of the spectrum $(600-1020 \mathrm{~nm}, 2.5 \mathrm{~nJ},<6 \mathrm{fs})$ seeds the NOPA. Optical seeding of the fiber pump laser intrinsically synchronizes pump and seed in the NOPA stages. The fiber pump laser chain, CPA-based, mainly consists of two Yb-doped photonic crystal rod-type fiber amplifiers which provide 11 and $15 \mathrm{~W}$ IR-average power, respectively (see Fig. 1). The fiber output pulses are frequency doubled to $515 \mathrm{~nm}$ and pump two NOPA-stages consisting of two $5 \mathrm{~mm}$ long Type-I BBO crystals configured for Poynting-VectorWalk-off Compensation (PVWC) geometry [19]. The signal from the oscillator is stretched to match the pump pulse duration, amplified into the two NOPA-stages, and finally compressed to below 7 fs using chirped mirrors. The output beam has an energy of $10 \mu \mathrm{J}$ per pulse at $200 \mathrm{kHz}$ repetition rate.

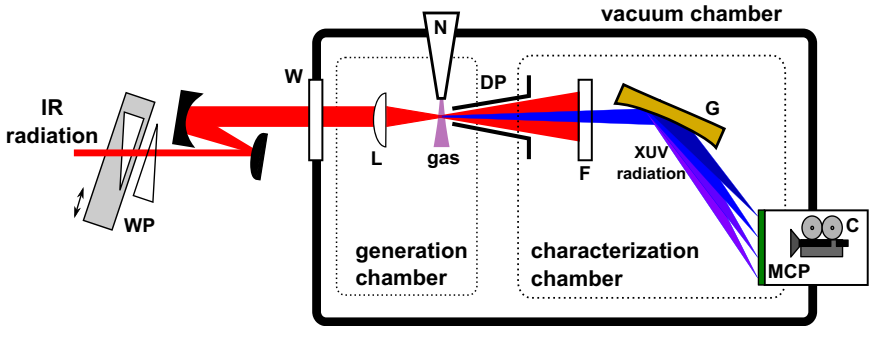

Fig. 2. Experimental setup for HHG including a wedge pair (WP), a telescope, and a vacuum chamber composed of a generation chamber and a characterization chamber hosting the XUV spectrometer. WP - wedges, W - window, L - lens, $\mathrm{N}$ - gas nozzle, DP - differential pumping hole, $\mathrm{F}$ - filter, $\mathrm{G}$ - grating, MCP - multiphoton channel plate, $\mathrm{C}$ - camera.

\section{Experimental setup: HHG}

The CEP-stable pulses from the OPCPA are sent into the HHG setup, illustrated in Figure 2. The setup comprises two chambers: one for the generation and one for the detection of XUV radiation, separated from each other by a small conical expanding hole for differential pumping. Thus, the pressure in the generation chamber can be as high as $10^{-2}$ mbar (with gas load), while the pressure in the detection chamber remains below $10^{-7}$ mbar.

The pulses from the OPCPA are negatively chirped to pre-compensate additional dispersion of optical components used in the HHG setup, i.e. the vacuum window and the focusing lens. Fine tuning of the dispersion is carried out by a pair of BK7, anti-reflection coated wedges mounted on a motorized translation stage. After the wedge-pair, the beam propagates through an allreflective telescope, where the beam diameter is expanded from 2 to $5 \mathrm{~mm}$. Afterwards, it enters the vacuum chamber through a $0.5 \mathrm{~mm}$-thick anti-reflection coated window. The fundamental beam is focused with an achromatic lens 


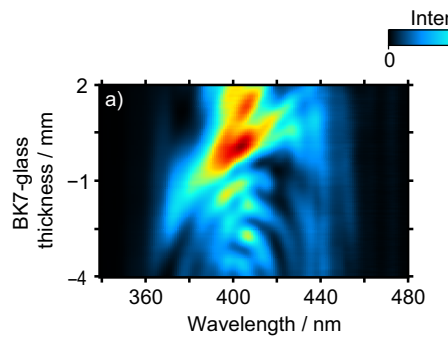

Intensity / arb. u.
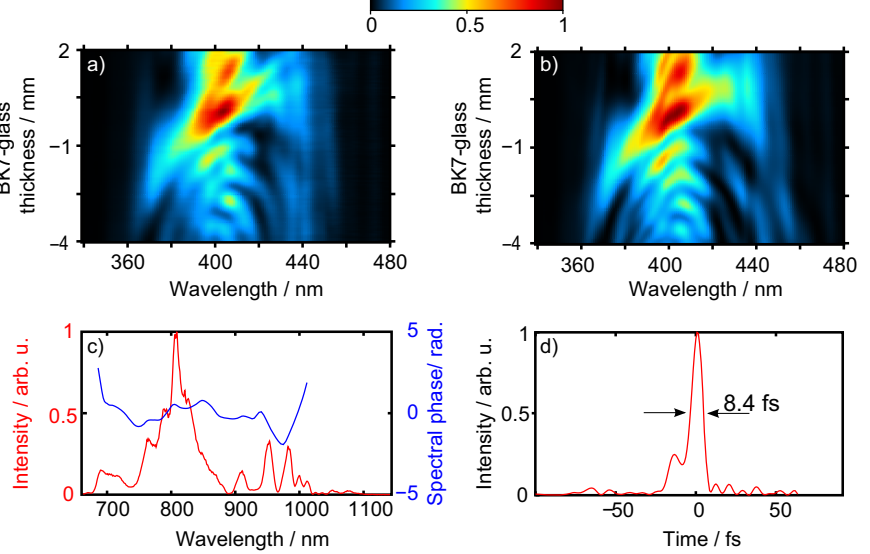

Fig. 3. Temporal characterization of the pulse after the lens with d-scan. (a) Measured and (b) retrieved d-scan traces. (c) Reconstructed spectral phase of the IR-pulse (blue) and measured spectral intensity of the pulse (red). (b) Reconstructed temporal pulse (for zero BK7-glass thickness) with a full width at half maximum of $8.4 \mathrm{fs}$.

$(f=5 \mathrm{~cm})$ into an effusive Ar gas jet. Due to the large bandwidth of the pulses a small effect of chromatic aberration remains. According to the design data of the lens, the position of the focus varies by about $60 \mu \mathrm{m}$ within a wavelength range from 650 to $1000 \mathrm{~nm}$, which is comparable to the estimated Rayleigh length $(50 \mu \mathrm{m})$.

Figure 3 shows the spectral phase of the pulse obtained from a dispersion-scan measurement (d-scan) after the lens and its reconstructed temporal pulse profile. The d-scan technique [20] is based on modifying the pulse spectral phase by propagation through wedges, frequency doubling and measuring the obtained spectra as a function of dispersion (propagation distance in the wedges). The spectral phase is then iteratively reconstructed from the dispersion scan. With the central wavelength of $890 \mathrm{~nm}$ and a measured pulse duration of $8.4 \mathrm{fs}$ the pulse includes three optical cycles under its full width at half maximum. The achromat was chosen mostly for the benefit of simple alignment. Moreover, obvious alternatives to focus the beam, i.e. spherical mirrors and metallic off-axis parabolic mirrors, were tried, but ruled out by either the strong astigmatism of short focal length spherical mirrors or by the often poor surface quality of metallic off-axis parabolas. In the future, a custom-made, dielectric focusing parabola might be the solution to obtain a diffraction limited spot size without impact on the pulse duration.

The Ar gas jet originates from a $90 \mu \mathrm{m}$ diameter nozzle, with 4 bar backing pressure. The nozzle is positioned as closely as possible to the laser beam by using a threeaxis translation stage. Tight focusing results in a short Rayleigh length, a small interaction volume, and a steep Gouy-phase gradient with consequences for HHG phase matching [21]. In order to compensate for both small volume and steep Gouy-phase gradient, a high generation gas density is needed. Focusing the fundamental beam with an $f$-number around 10 implies that a generation pressure of a few bars is needed to achieve phase matching $[22,23]$.

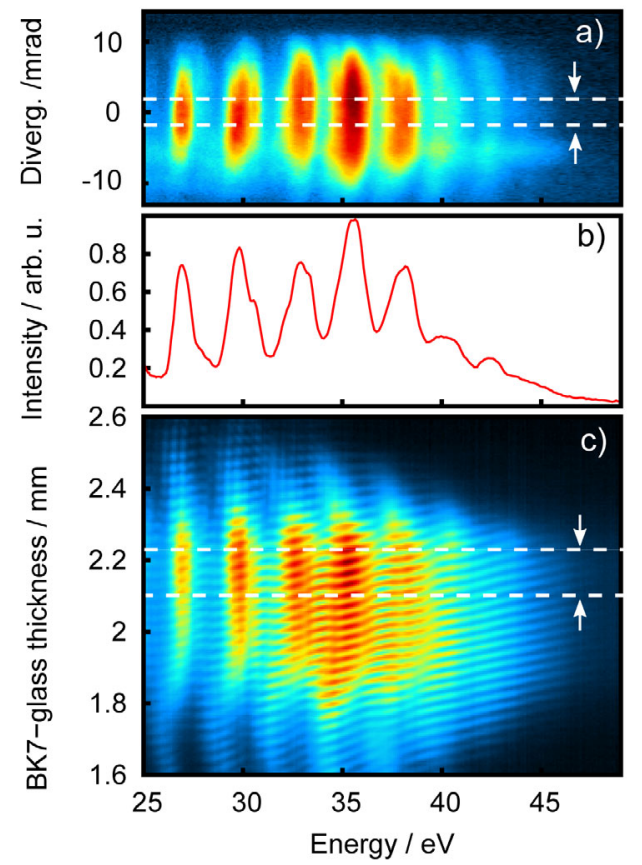

Fig. 4. (a) Spatially-resolved HHG spectrum recorded by imaging the MCP phosphor screen with a camera. The integration of the spatial profile (using only the marked region) leads to the HHG spectrum shown in (b). (c) Integrated HHG spectrum vs. BK7 wedge thickness. The influence of the change of the carrier-envelope phase over the whole HHG spectrum is clearly visible as well as the influence of the material dispersion on the IR pulse duration.

The gas pressure used in the present setup is limited by the pumping system and is estimated to be below 1 bar in the interaction region. Thus, high-order harmonics are not generated fully phase-matched. The gas target is located behind the laser focus, so that the short trajectory contribution to HHG is selected [24].

The generated high-order harmonic radiation and the fundamental IR laser pulses enter the characterization chamber through the differential pump hole. The HHG beam can be separated from the fundamental by metallic filters, e.g. $200 \mathrm{~nm}$-thick aluminum or titanium filters, installed in a motorized filter mount. The XUV radiation is recorded with a spectrometer consisting of a cylindrical diffraction grating (Hitachi, 600 lines/mm), a Micro Channel Plate (MCP), and a phosphor screen. The MCP is coated with a CsI layer in order to extend its sensitivity range in the VUV spectral range. Spatially-resolved harmonic spectra are recorded by imaging the phosphor screen with a CCD camera. The spectra are calibrated in photon energy using the well known transmission edges of different filters [25].

\section{Results}

Figure 4a shows a typical, spatially-resolved harmonic spectrum generated in Ar in the photon energy range from 25 to $45 \mathrm{eV}$. A spectrum integrated over the central region (indicated by the dashed white lines) is presented in 

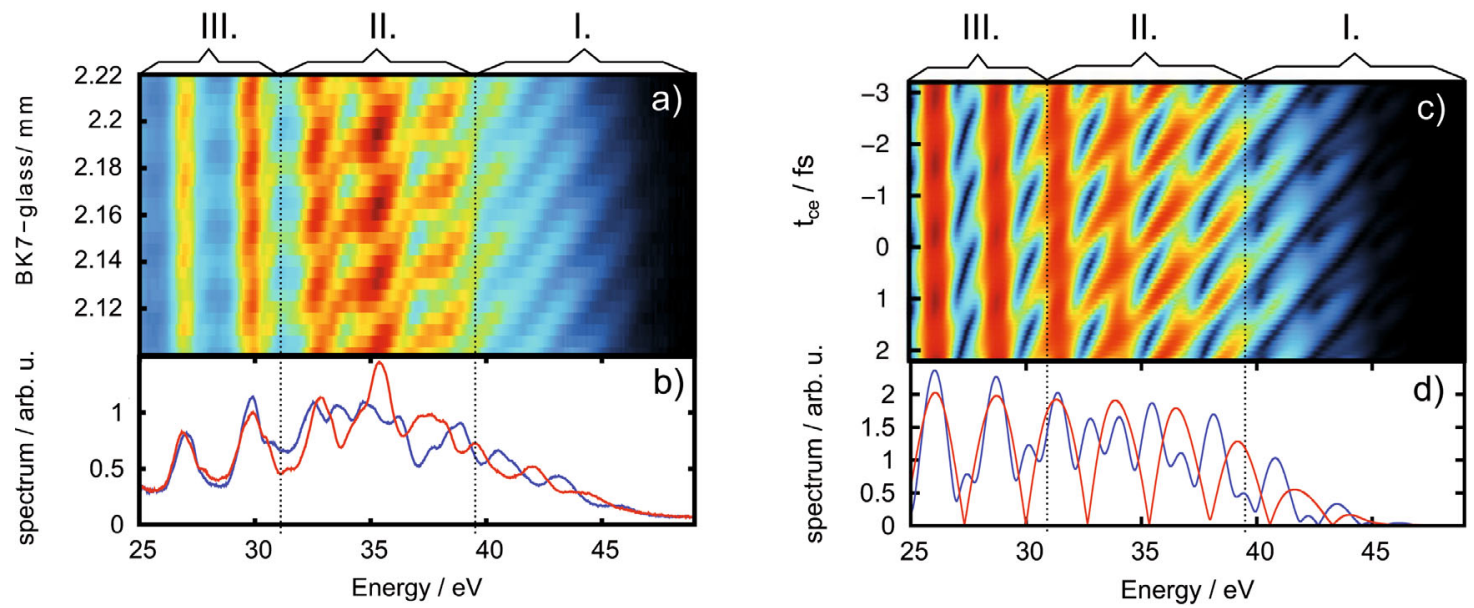

Fig. 5. Measured (a) and simulated (c) HHG spectra as a function of the carrier-envelope phase of the driver. Corresponding to (a) and (c), (b) and (d) present two lineouts with a change of the CEP of $\Delta \varphi=\pi / 2$ between them. Three regions are marked in the figures: regions I, II and III, which indicate different types of CEP dependence.

Figure 4b. The harmonics are spectrally broad, especially in the cut-off region above $40 \mathrm{eV}$.

Figure 4c presents the integrated HHG spectrum as a function of the wedge thickness (see wedge pair in Fig. 2). Translating one wedge with respect to the other changes both the carrier-envelope phase of the IR-pulses and the overall dispersion of the pulse, affecting the pulse duration and the intensity. Such a scan takes less than a minute, and laser instabilities are therefore negligible. A change of thickness of $\sim 30 \mu \mathrm{m}$ BK7 glass shifts the carrier-envelope phase by $\pi$, while the simultaneous change in pulse duration is negligible. The scan shown in Figure $4 \mathrm{c}$ spans over a variable glass thickness of $1 \mathrm{~mm}$. Both CEP and intensity effects are clearly visible. Below 1.8 and above $2.4 \mathrm{~mm}$ the pulse is considerably chirped and the laser intensity is too low to efficiently generate high-order harmonics. HHG is efficient within a range of about $0.5 \mathrm{~mm}$ of BK7, corresponding to the shortest pulses. Most remarkable in the recorded HHG spectra is the strong CEP dependence, which is not limited to the cut-off region, but is clearly observable in the presented spectrum.

The behavior seen in our experiment is shown in more detail in Figure 5a (a zoom to the area between the dashed, white lines in Fig. 4c). The plotted range corresponds to a change of BK7-glass thickness of $120 \mu \mathrm{m}$; the impact on the change of pulse duration can be neglected, CEP effects clearly dominate. Figure $5 \mathrm{~b}$ presents two lineouts from Figure 5a with a change of CEP of $\Delta \varphi=\pi / 2$ between them. Three different regions can be distinguished: in region $\mathrm{I}$, in the cut-off (above $40 \mathrm{eV}$ ), spectral peaks shift from odd to even harmonics as the CEP changes by $\pi / 2$. In region III (low harmonic orders), the position of the harmonic peaks does not change, but their amplitude depends on the CEP. For example, the 17 th and 19th harmonics reach a maximum for different CEP values. In between, in region II, the harmonic spectrum shows non trivial dynamics upon CEP change; spectral peaks are not located at multiple orders of the laser frequency and their position shift with CEP.

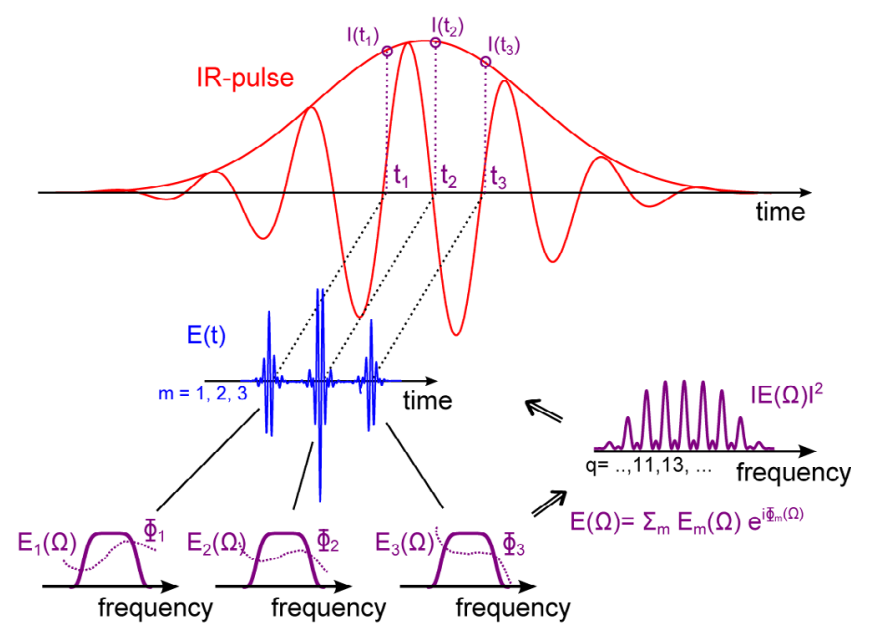

Fig. 6. Sketch of the multipulse interference mode. A IR driving pulse generates three attosecond pulses leading to an attosecond pulse train. Every attosecond pulse in time can be described with a spectral amplitude and phase in the Fourier domain. The coherent superposition of the three complex spectra leads to the high-order harmonic spectrum $E(\Omega)$.

\section{Discussion}

To interpret the complex spectra shown in Figure 5a, we develop a model based upon interferences of multiple pulses. A Fourier transformation of the spectra, assuming a constant spectral phase, indicates that the number of pulses in the train is approximately three, as illustrated in Figure 6. The next step consists in coherently summing the complex spectra of these attosecond pulses, taking into account the CEP-dependent spectral phase,

$$
E(\Omega)=\sum_{m=1}^{3} E_{m}(\Omega) e^{i \Phi_{m}(\Omega)},
$$

with

$$
\Phi_{m}(\Omega)=m \pi+\Omega t_{m}+\alpha_{\Omega} I\left(t_{m}\right) .
$$


The first term comes from the symmetry of HHG: two consecutive pulses are opposite in sign. In the second term, $t_{m}$ indicates the (periodic) timing of the XUV attosecond pulses in the train. Two consecutive pulses are separated in time by half a laser period: $t_{m+1}-t_{m}=T / 2$. Finally the third term reflects the influence of the generation process with a phase contribution which depends on the driving laser intensity at $t_{m}$ [26]. This term will lead to a phase variation of the attosecond pulse as well as to a slight change of timing of the attosecond pulses (not included in $t_{m}$ ). The laser profile is assumed to be Gaussian with duration $\tau=7$ fs and peak intensity $I_{0}=1.5 \times 10^{14} \mathrm{~W} / \mathrm{cm}^{2}$.

Figure $5 \mathrm{~b}$ shows $|E(\Omega)|^{2}$ as a function of the laser CEP and $\Omega$. In our model, the laser CEP influences the timing of the attosecond pulses in the train and therefore, for short enough pulses, the spectral phase which depends on $I\left(t_{m}\right)$. The experimental results are well reproduced in all three spectral regions. To understand better how our simple model (Eq. (2)) leads to the interference structure shown in Figure 5b, we calculate the phase difference between two consecutive attosecond pulses:

$$
\Delta \Phi(\Omega)=\pi+\frac{\Omega T}{2}+\alpha_{\Omega}\left[I\left(t_{1}+\frac{T}{2}\right)-I\left(t_{1}\right)\right] .
$$

For long IR pulses, $I\left(t_{1}+T / 2\right) \approx I\left(t_{1}\right)$, so that $\Delta \Phi \equiv 0$ $(\bmod 2 \pi)$ when $\Omega=q \omega$, where $q$ is an odd integer. Destructive interferences lead to the extinction of the XUV emission at all frequencies except those at odd multiple orders of the laser frequency and the CEP does not affect the HHG spectrum. For short pulses, the laser intensity changes between consecutive half cycles and the interference pattern becomes more complex. By Taylor expanding $I(t)$ around $t=0$, equation (3) becomes

$$
\Delta \Phi(\Omega) \approx \pi+\frac{\Omega T}{2}-\frac{4 \ln 2 T}{\tau^{2}}\left(t_{1}+\frac{T}{4}\right) \alpha_{\Omega} I_{0} .
$$

We can relate $t_{1}$, or equivalently $t_{1}+T / 4$ to the carrierenvelope phase of the IR field through the equation $t_{1}+T / 4=\phi_{c e} T / 2 \pi . \phi_{c e}$ represents the phase difference between $t_{1}+T / 4$ and the pulse center (We are only concerned here with the relative value of the CEP). Equation (4) becomes

$$
\Delta \Phi(\Omega) \approx \pi+\frac{\Omega T}{2}-\frac{2 \ln 2 T^{2} \phi_{c e} \alpha_{\Omega} I_{0}}{\pi \tau^{2}} .
$$

It is interesting to consider the variation of $\Delta \Phi$ with respect to $\phi_{c e}$.

$$
\frac{\partial \Delta \Phi}{\partial \phi_{c e}}=-\frac{2 \ln 2 T^{2} \alpha_{\Omega} I_{0}}{\pi \tau^{2}} .
$$

For long pulses, low intensities or low photon energy such that $\alpha_{\Omega}$ is small [27], $\partial \Delta \Phi / \partial \phi_{c e} \approx 0$, which implies that the position of the harmonics does not depend on the CEP (region III). If $\partial \Delta \Phi / \partial \phi_{c e} \approx 1, \Delta \Phi$ varies by $\pi$ when $\phi_{c e}$ changes by $\pi$ and the position of the harmonic peaks moves from an odd to an even multiple of the laser frequency (region I). More generally, when $\partial \Delta \Phi / \partial \phi_{c e} \neq 0$ $(\bmod 2)$, the position of the harmonic peaks will move with CEP. This variation increases with frequency as $\alpha_{\Omega}$ increases towards the cutoff. When $\partial \Delta \Phi / \partial \phi_{c e}$ is not close to an integer, the interference pattern will exhibit discontinuities as the CEP is varied and the spectrum will have a complex behavior as is the case in region II.

\section{Summary}

This article presents studies of high-order harmonic generation, driven by a high-repetition rate OPCPA laser system. In spite of the low available IR energy per pulse $(10 \mu \mathrm{J})$, there is no difficulty in generating harmonics in Ar. This is achieved in a tight focusing geometry and with high generation pressure. Many applications in attosecond science should benefit from attosecond sources operating at higher repetition rates, i.e. in the $\mathrm{MHz}$ range [22,28-32]. Examples of such applications are coincidence detection of two or more particles, which require a low number of events per laser shot [33], but a large number of events per second to build up statistics, photoelectron spectroscopy and microscopy in solids and on solid surfaces $[34,35]$ where space charge effects can be a serious obstacle.

The excellent CEP-stability of the system allows us to study in detail the HHG spectrum as a function of the CEP of the driving pulses. We find that the HHG spectrum strongly depends on the CEP, over the whole spectral range from 25 to $45 \mathrm{eV}$. In the cutoff region, the harmonic peaks shift from odd to even orders, while at low energy, the CEP only affects the amplitudes of the peaks. In an intermediate region between 32 and $40 \mathrm{eV}$, the spectra becomes nontrivial and the number of spectral peaks increases. We developed a simple model which reproduces well the experimental observations. Our interpretation is that in our experiment, the spectra are affected by the CEP-dependence of the spectral phase difference between contributing attosecond pulses. We also provide an analytical derivation of the structure of the interference pattern. Precise CEP-dependent HHG spectra could be used in the future to get information on the atomic or molecular systems used for the generation, which would be extremely useful e.g. for tomographic reconstruction.

This research was supported by the Marie Curie Research Training Network ATTOFEL, the European Research Council (ALMA, PALP), the Knut and Alice Wallenberg foundation, the Swedish Foundation for Strategic Research, and the Swedish Research Council.

P. Rudawski and A. Harth contributed to this work equally.

\section{References}

1. A. Dubietis, G. Jonušauskas, A.P. Piskarskas, Opt. Commun. 88, 437 (1992)

2. A. Vaupel, N. Bodnar, B. Webb, L. Shah, M. Richardson, Opt. Eng. 53, 051507 (2013) 
3. D. Herrmann, L. Veisz, R. Tautz, F. Tavella, K. Schmid, V. Pervak, F. Krausz, Opt. Lett. 34, 2459 (2009)

4. M. Schultze, T. Binhammer, G. Palmer, M. Emons, T. Lang, U. Morgner, Opt. Express 18, 27291 (2010)

5. A. Harth, M. Schultze, T. Lang, T. Binhammer, S. Rausch, U. Morgner, Opt. Express 20, 3076 (2012)

6. J. Matyschok, T. Lang, T. Binhammer, O. Prochnow, S. Rausch, M. Schultze, A. Harth, P. Rudawski, C.L. Arnold, A. L'Huillier, U. Morgner, Opt. Express 21, 29656 (2013)

7. R. Riedel, A. Stephanides, M. Prandolini, B. Gronloh, B. Jungbluth, T. Mans, F. Tavella, Opt. Lett. 39, 1422 (2014)

8. J. Nillon, O. Crégut, C. Bressler, S. Haacke, Opt. Express 22, 14964 (2014)

9. J. Rothhardt, S. Demmler, S. Hädrich, J. Limpert, A. Tünnermann, Opt. Express 20, 10870 (2012)

10. M. Krebs, S. Hädrich, S. Demmler, J. Rothhardt, A. Zaïr, L. Chipperfield, J. Limpert, A. Tünnermann, Nat. Photon. 7, 555 (2013)

11. S. Hädrich, A. Klenke, J. Rothhardt, M. Krebs, A. Hoffmann, O. Pronin, V. Pervak, J. Limpert, A. Tünnermann, Nat. Photon. 8, 779 (2014)

12. A. Baltuška, Th. Udem, M. Uiberacker, M. Hentschel, E. Goulielmakis, Ch. Gohle, R. Holzwarth, V.S. Yakovlev, A. Scrinzi, T.W. Hänsch, F. Krausz, Nature 421, 611 (2003)

13. E. Mansten, J.M. Dahlström, J. Mauritsson, T. Ruchon, A. L'Huillier, J. Tate, M.B. Gaarde, P. Eckle, A. Guandalini, M. Holler, F. Schapper, L. Gallmann, U. Keller, Phys. Rev. Lett. 102, 083002 (2009)

14. M. Nisoli, G. Sansone, S. Stagira, S. De Silvestri, C. Vozzi, M. Pascolini, L. Poletto, P. Villoresi, G. Tondello, Phys. Rev. Lett. 91, 213905 (2003)

15. G. Sansone, C. Vozzi, S. Stagira, M. Pascolini, L. Poletto, P. Villoresi, G. Tondello, S. De Silvestri, M. Nisoli, Phys. Rev. Lett. 92, 113904 (2004)

16. G. Sansone, E. Benedetti, J.-P. Caumes, S. Stagira, C. Vozzi, M. Pascolini, L. Poletto, P. Villoresi, S. De Silvestri, M. Nisoli, Phys. Rev. Lett. 94, 193903 (2005)

17. C. Ott, M. Schönwald, P. Raith, A. Kaldun, G. Sansone, M. Krüger, P. Hommelhoff, Y. Patil, Y. Zhang, K. Meyer, M. Laux, T. Pfeifer, New J. Phys. 15, 073031 (2013)

18. S. Rausch, T. Binhammer, A. Harth, F.X. Kärtner, U. Morgner, Opt. Express 16, 17410 (2008)

19. T. Lang, A. Harth, J. Matyschok, T. Binhammer, M. Schultze, U. Morgner, Opt. Express 21, 949 (2013)

20. M. Miranda, T. Fordell, C. Arnold, A. L'Huillier, H Crespo, Opt. Express 20, 688 (2012)

21. A. L'Huillier, X.F. Li, L.A. Lompré, J. Opt. Soc. Am. B 7, 527 (1990)
22. C.M. Heyl, J. Güdde, A. L'Huillier, U. Höfer, J. Phys. B 45, 074020 (2012)

23. J. Rothhardt, M. Krebs, S. Hädrich, S. Demmler, J. Limpert, A. Tünnermann, New J. Phys. 16, 033022 (2014)

24. P. Salières, A. L'Huillier, M. Lewenstein, Phys. Rev. Lett. 74, 3776 (1995)

25. B.L. Henke, E.M. Gullikson, J.C. Davis, At. Data Nucl. Data Tables 54, 181 (1993)

26. M. Lewenstein, K.C. Kulander, K.J. Schafer, P.H. Bucksbaum, Phys. Rev. A 51, 1495 (1995)

27. K. Varjú, Y. Mairesse, B. Carre, M.B. Gaarde, P. Johnsson, S. Kazamias, R. Lopez-Martens, J. Mauritsson, K.J. Schafer, Ph. Balcou, A. L'Huillier, P. Salières, J. Mod. Opt. 52, 379 (2005)

28. J. Boullet, Y. Zaouter, J. Limpert, S. Petit, Y. Mairesse, B. Fabre, J. Higuet, E. Mével, E. Constant, E. Cormier, Opt. Lett. 34, 1489 (2009)

29. C. Chiang, A. Blättermann, M. Huth, J. Kirschner, W. Widdr, Appl. Phys. Lett. 101, 071116 (2012)

30. O.H. Heckl, C.R.E. Baer, C. Kränkel, S.V. Marchese, F. Schapper, M. Holler, T. Südmeyer, J.S. Robinson, J.W.G. Tisch, F. Couny, P. Light, F. Benabid, U. Keller, Appl. Phys. B 97, 369 (2009)

31. F.J. Furch, S. Birkner, F. Kelkensberg, A. Giree, A. Anderson, C.P. Schulz, M.J.J. Vrakking, Opt. Express 21, $22671(2013)$

32. E. Lorek, E.W. Larsen, C.M. Heyl, S. Carlström, D. Paleček, D. Zigmantas, J. Mauritsson, Rev. Sci. Instrum. 85, 123106 (2014)

33. E.P. Månsson, D. Guénot, C.L. Arnold, D. Kroon, S. Kasper, J.M. Dahlström, E. Lindroth, A.S. Kheifets, A. L'Huillier, S.L. Sorensen, M. Gisselbrecht, Nat. Phys. 10, (2014)

34. T. Haarlammert, H. Zacharias, Curr. Opin. Solid State Mater. Sci. 13, 13 (2009)

35. A. Mikkelsen, J. Schwenke, T. Fordell, G. Luo, K. Klünder, E. Hilner, N. Anttu, A.A. Zakharov, E. Lundgren, J. Mauritsson, J.N. Andersen, H.Q. Xu, A. L'Huillier, Rev. Sci. Instrum. 80, 123703 (2009)

Open Access This is an open access article distributed under the terms of the Creative Commons Attribution License (http://creativecommons.org/licenses/by/4.0), which permits unrestricted use, distribution, and reproduction in any medium, provided the original work is properly cited. 